\title{
Collaborative Social Bidding Decision-making Mechanism of Service Recommendation
}

\author{
Xiaona Xia ${ }^{1+}$, Jiguo $\mathrm{Yu}^{1}$, Lianyong $\mathrm{qi}^{1}$, Jianchao $\mathrm{Zheng}^{1}$, Lu Zhang ${ }^{1}$ \\ ${ }^{1}$ School of Information Science and Engineering, Qufu Normal University, Rizhao, China
}

\begin{abstract}
Promotion of network services and social service systems, how to recommend appropriate services to goal users becomes a key problem. This paper clusters users and services in social service system respectively, achieves social circle and service cluster models, based on, we design service recommendation mechanism with social bidding decision-making and specific implementation algorithm, constructs service recommendation process driven by demand preferences. Through the experiment, we can realize accuracy and effectiveness of decision-making, and improve the efficiency and quality of service recommendation.
\end{abstract}

Keywords: Service Recommendation, Service Cluster, Social Circle, Bidding Pattern, Decision-making; Demand Preferences

\section{Introduction}

The emergence of massive services in internet expands the network functions and potential abilities[1,2], and also brings new problems. Social network services (SNS (Social Network Service) is becoming more and more mature, how to discover, select and use this services in order to cope with the change of demands, that brings new challengers. The goal of service users is to find and use satisfied services in the shortest possible time, and to complete the results feedback [3].

Service recommendation is one of the most effective methods to solve overload phenomenon, collaborative filtering is the most effective method, but it is inefficient for the cold start of services [4]. As is known to all, in the social network, users selection will be affected by their friends, the occurred interest preferences and services implementation might bring consideration for references [5]. The implementation of historical services of social circles achieve decision-making selection and recommendation, which has a strong research significance and application prospects [6].

Social Recommendation constructs social relationship among users. For one new user, as long as there are neighbors or friends, we can deduce the potential interest preferences, whose recommendation strategy is reasonable and effective, which results from the important research results of social network[7, 8].

\section{Related Work}

Service recommendation selects appropriate services to meet the demand interests of users, corresponding social information is the important factor, service recommendation with social information and decision-making ability can help users to discover high quality services. [9] innovated active learning and decision-making, designed one active learning framework of service decision-making, rated and ranked service applications of services. [10] designed multi-view deep learning methods of inter domains, by reducing the number of input dimension and training set, extended user models based on complete features were achieved. [11] quantified effectiveness of long-term models and short-term adaptive strategies. [12] analyzed two kinds of machine learning methods to predict the best execution services. The first method

\footnotetext{
${ }^{+}$Corresponding author. Tel.: +8613561927395; fax: +86-0633-3980242.
}

E-mail address: xiaxn@sina.com. 
focused on classification, the second focused on regression and predicted service performances to determine the best candidate projects. [13] predicted online recommendation performances by offline performances, constructed performance models, balanced different performances, and adjusted optimal recommendation performances. [14] proposed Distributed semantic pre-filtering method, built accurate perception rating prediction models. [15] proposed the latent feature model of multi dimension group based on time perception to improve the accuracy of recommendation. [16] used machine learning reasoning methods to recommend services. [17] extended predicted ordering based on hybrid recommendation, designed PGraph to describe neighbor services. [18] considered time dynamic of QoS and individual factors of users to achieve prediction about collaborative filtering and time series.

\section{Social Demand and Service Clustering}

\subsection{Social demand}

Social recommendation system involves two relationship models: (1) social relationship graph, nodes of graph represent users, the connections between nodes represent social relationships. We establish evaluation criteria and reasoning rules to measure the degree of social interactions. (2) user-item rating network topology. Different recommendation technologies are corresponding to different network graph topology. At present, network topology is static and single, which rarely involves the dynamic nature of user demands and multi layer problems of network. Based on the first approach, this paper proposes bidding decision-making of social relationship topology to achieve the recommended evaluation and reasoning process. Fig. 1 is social service recommendation architecture.

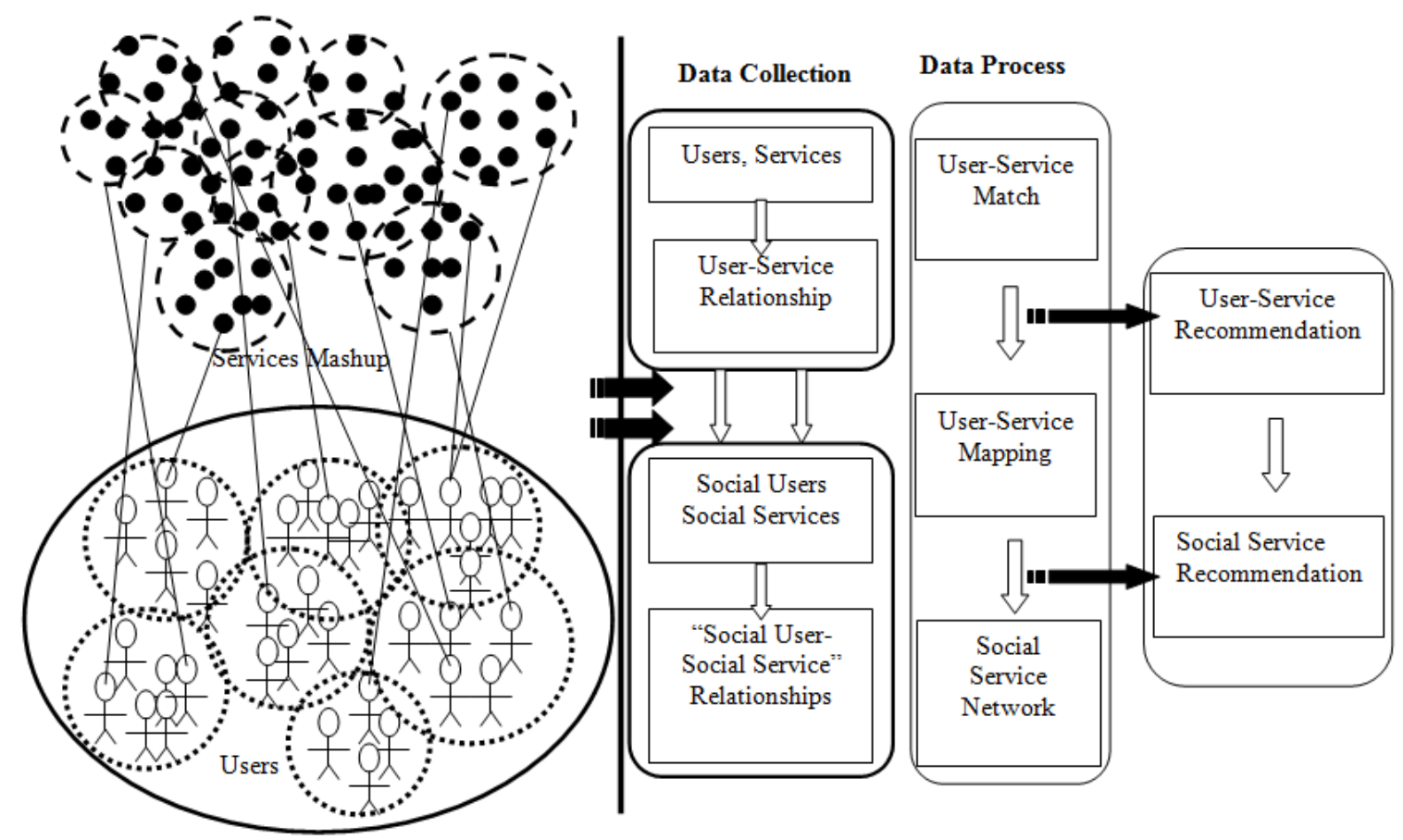

Fig. 1. Social service recommendation process

\subsection{Service search rules}

In social service recommendation system, service satisfaction determines service recommendation and selection, the relevant rules of service satisfaction are given below.

Definition 1 Supposing $T_{1}, T_{2} \cdots T_{m}$ are the service list of $\operatorname{tag}_{1}, \operatorname{tag}_{2} \ldots \operatorname{tag}_{m}$, $T_{1} \cap T_{2} \cdots T_{m}=\left\{<s_{i},\left(p_{p} p_{2}, \cdots, p_{m}\right) \ngtr<s_{i}, p_{1}>\in T_{1},<s_{i}, p_{2}>\in T_{2}, \ldots,<s_{i}, p_{m}>\in T_{m}\right\}$, if its result is not null, the elements are atomic services to satisfy $\operatorname{tag}_{1}, \operatorname{tag}_{2} \ldots \operatorname{tag}_{m}$. 
Definition 2 Supposing $T_{1}, T_{2} \cdots T_{m}$ are the service list of $\operatorname{tag}_{1}, \operatorname{tag}_{2} \ldots \operatorname{tag}_{m}$, $\left.T_{1} \times T_{2} \cdots T_{m}=\left\{<\left(s_{1}, s_{2}, \cdots, s_{m}\right), p_{1}, p_{2}, \cdots, p_{m}\right\rangle<s_{1}, p_{1}\right\rangle$, the elements in set is the service cluster to $\left.\in T_{1},<s_{2}, p_{2}>\in T_{2}, \cdots,<s_{m}, p_{m}>\in T_{n}\right\}$ satisfy $\operatorname{tag}_{1}, \operatorname{tag}_{2} \operatorname{tag}_{m}$, the service cluster can satisfy one or more $\operatorname{tag}$.

\section{Bidding Decision-Making Principle}

Users and services of social network need collaborative decision-making, the bidding process of service clusters and social circles needs to consider computational complexity, QoS, subjective trust and service satisfaction, but these factors do not directly for goal bidding and selecting. We define relationship organization of decision-making goal of service bidding:

Decomposition: according to the functions of service clusters and $Q o S, g_{i}$ is decomposed into a set of sub goals $S G^{d}=\left(g_{i 1}, g_{i 2}, \ldots, g_{i m}\right), S G . g_{i j} \propto g_{i}$, and there is a "and" relationship between the sub goals.

Substitution: when any goal in $S G^{t}=\left(g_{i}{ }^{1}, g_{i}^{2}, \ldots, g_{i}^{n}\right)$ has been completed, $g_{i}$ is realized, that is expressed as $S G . g_{i}^{j} \equiv g_{i}$, there is "or" relationship between the sub goals.

Atomic: one goal without any sub goals is called an atomic goal.

With the help of "and" and "or" relationship of goals, we can construct the "and tree" or "or tree" structure of goals, $g$ is root node, the sub goal $g_{i j}$ of $S G^{d}$ is the sub node of $g_{i}$, the sub goal $g_{i}^{j}$ of $S G^{t}$ is the brother node of $g_{i}$, all leaf nodes are all atomic goals.

$g$ can be achieved by service set $S G$, iff the services corresponding to all leaf nodes of "and tree" of $g$ are all in $S G$, or can be replaced by the goals in $S G$.

\section{Optimized Recommendation Algorithm Based on Decision-making}

We firstly give goal evaluation strategy of social cooperation decision-making bidding, according to semantic definition of the forth part of this paper, that is mainly related to the two aspects, pre-goal and need.

According to goal and $s T p$, The evaluation process of pre-goal is described as Algorithm 1.

\begin{tabular}{|c|c|}
\hline Algorithm 1: The evaluation process of pre-goal & Algorithm 2: Bidding Decision-making Process of Social Service Recommendation \\
\hline Get goal.or-tree & $C=\phi, D=\phi$, pre - goal $/ /$ Candidate service set is initialized null, bidding decision-making \\
\hline If $g_{i} \in$ pre - goal $\wedge g_{i} \equiv$ goal & set is initialized null. \\
\hline Then $u(\text { pre }- \text { goal })_{t e \varepsilon}=1$ & Set( pre - goal $) / /$ Set initial demand target \\
\hline Return $1 / /$ end Evaluation & For $D e \in$ Decision - List do \\
\hline Else get goal.and-tree & If $\mid u(d e)-u($ pre - goal $) \mid>\sigma / / \sigma \in[0,1]$, Calculate the approximate threshold of target \\
\hline If pre-goal $\propto$ goal & Then $C=C \cup\{D e\}$, send (De.tender $\operatorname{Pr}$ ofile) $/ /$ Send bidding request \\
\hline Then $u(\text { pre }- \text { goal })_{\text {tree }}=1$ & For $D e \in C$ do \\
\hline Return $1 / /$ end Evaluation & If accept tender Profile $\left.\right|_{i}$ \\
\hline Else while $s u b-$ goal $_{j} \in$ goal do & Then send (tend $\operatorname{Pr}$ ofile $i$ id, Accept) \\
\hline 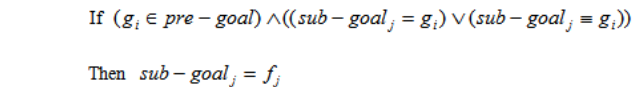 & $D=D \bigcup\{D e\}$ \\
\hline & Else return//Abandon Bidding \\
\hline$u(\text { pre }-g o a l)_{t e \varepsilon}=\sum w f_{i} / /$ get Evaluation value & End \\
\hline
\end{tabular}

Service recommendation algorithm completes bidding process of demands and service, social circle invites demands and define decision-making interactive list Decision - List and records interactive historical information of social circle services. Based on social relationships, we expands the scope of service tender, the whole process is described as Algorithm 2.

\section{Case Analysis and Experimental Results}

We statement accuracy of bidding decision-making based on user number $=3500$ and service number $=17000$, clustering users to form social circles, clustering services to form configuration service 
clusters. Setting different conditions, this experiment is divided into three steps, the first step contains the first group and the forth group, before bidding, we set the most bidding ability. The second step contains contains the third group and the fifth group, we set the reliable bidding ability and stable historical demand preferences. The third step contains the third group and the sixth group, it user Algorithm 2, based on social user cooperation and service clusters, we introduce user attributes and service characters. This experiment includes two conditions, one is Certainty of current demand goals and stability of service parameters (corresponding to the first, the second and the third group). Two is uncertainty of current demand goals and instability of services, including of new users and new services (corresponding to the forth, the fifth and the sixth group). These two conditions can be achieved by randomly setting the parameters of the users and the services. The scales of service clusters are set to 10,20,30, 40 and 50, the experimental results are as shown in Fig. 2, we can see, with the increase in the number of unstable factors, all kinds of bidding accuracy rates decline, the method of this paper can effectively guarantee the bidding accuracy, and the results are relatively better.
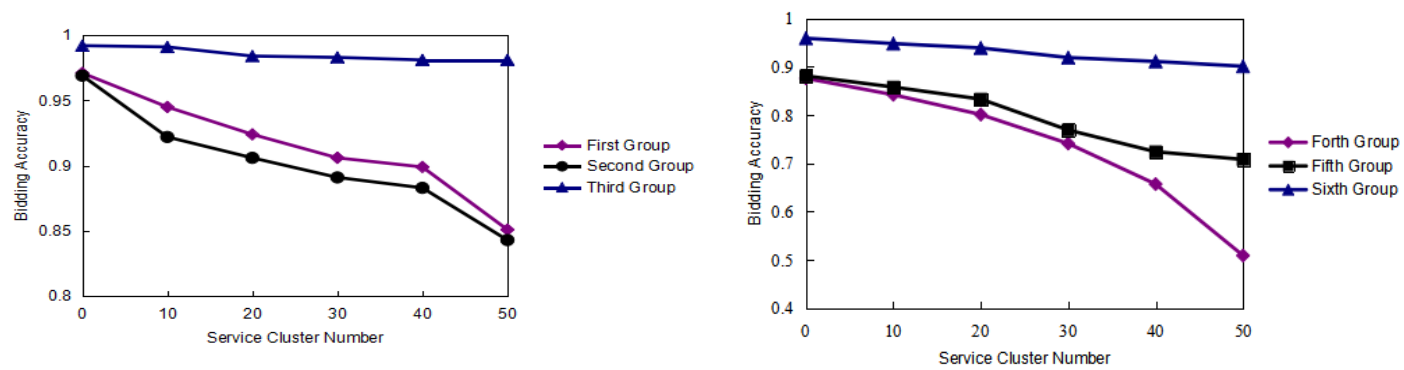

(1)Uncertainty of current demand preferences and instability of services (2) Uncertainty of current demand preferences and instability Fig. 2. Bidding accuracy comparison of service recommendation

Based on accuracy, we verify the validity of bidding decision-making. For 3500 users and 17000 services, we set 350 decision-making tasks, that is divided into three steps, the first step uses the service recommendation strategy of the first response and the first selection, the second step uses the service recommendation strategy with the most historical cooperation number, the third step uses Algorithm 3 and 4 of this paper. The experiment analyzes two aspects, (1) determination of current demand goals and stability of service function parameters(corresponding the first, second and third group); (2) the uncertainty of current demand goals and instability of service functions, including new users and new services(corresponding to the forth, fifth and sixth group), the experiment results are shown in (1) and (2) of Fig. 3, in the condition of ensuring the accuracy of bidding, we can guarantee the high decision evaluation rate.
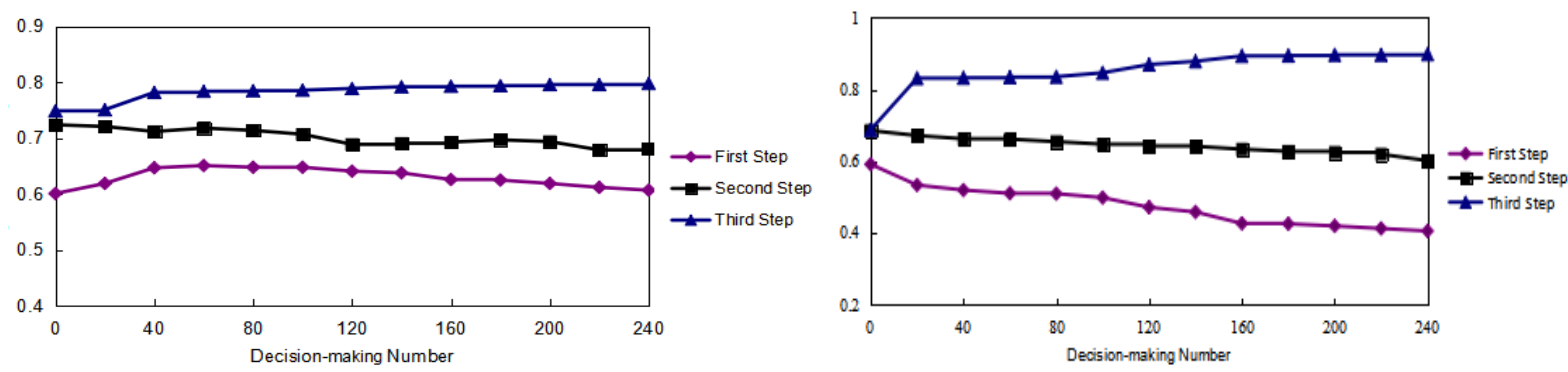

(1)Certainty of current demand preferences and stability of services (2)Uncertainty of current demand preferences and instability

Fig. 5 Bidding Effectiveness Comparison of Service Recommendation

\section{Conclusion}

Whether the function of atomic services or the cooperation of composition services, feedback information collection is difficult among users and services. In service recommendation, there are no effective methods to implement about user preference-driven service recommendation. From services and users, this paper puts forward social service recommendation mechanism based on service clustering and social cooperation. In recommendation system of massive services, we construct the clustering method of social circle and composite services, through user cooperation, we get historical demand preferences and potential demand preferences. Service clusters can achieve more service capabilities to meet more demand 
preferences. We build social bidding decision-making mechanism to achieve service recommendation between services and users.

\section{Acknowledgements}

This paper is supported by National Natural Science Foundation of China (61672321, 61373027, 61402258) and Natural Science Foundation of Shandong Province of China (ZR2016FM45, ZR2012FQ011).

\section{References}

[1] Lü L, Medo M, Yeung C H, et al. Recommender systems[J]. Physics Reports, 2012, 519(1): 1-49.

[2] Fletcher K K, Liu X F. A Collaborative Filtering Method for Personalized Preference-Based Service Recommendation[C]. Web Services (ICWS), 2015 IEEE International Conference on. IEEE, 2015: 400-407.

[3] Tang J, Zhang C, Cai K, et al. Sampling Representative Users from Large Social Networks[C]. 29th AAAI Conference on Artificial Intelligence. 2015.

[4] Kanoje S, Girase S, Mukhopadhyay D. User Profiling for Recommendation System[J]. arXiv preprint arXiv:1503.06555. 2015.

[5] Lee K, Lee K. Escaping your comfort zone: A graph-based recommender system for finding novel recommendations among relevant items[J]. Expert Systems with Applications. 2015, 42(10): 4851-4858.

[6] Bobadilla J, Ortega F, Hernando A, et al. Recommender systems survey[J]. Knowledge-Based Systems. 2013. 46: 109-132.

[7] Social Network Analysis. A brief introduction. 2007. http://orgnet.Com/sna.html

[8] MENG Xiang-Wu, LIU Shu-Dong, ZHANG Yu-Jie, HU Xun. Research on Social Recommender Systems[J]. Journal of Software,2015,26(6):1356-1372.

[9] Karimi R, Nanopoulos A, Schmidt-Thieme L. A supervised active learning framework for recommender systems based on decision trees[J]. User Modeling and User-Adapted Interaction, 2015, 25(1): 39-64.

[10] Elkahky A M, Song Y, He X. A Multi-View Deep Learning Approach for Cross Domain User Modeling in Recommendation Systems[C]. Proceedings of the 24th International Conference on World Wide Web. International World Wide Web Conferences Steering Committee. 2015: 278-288.

[11] Jannach D, Lerche L, Jugovac M. Adaptation and Evaluation of Recommendations for Short-term Shopping Goals[C]. Proceedings of the 9th ACM Conference on Recommender Systems. ACM, 2015: 211-218.

[12] Corbellini A, Mateos C, Godoy D, et al. An architecture and platform for developing distributed recommendation algorithms on large-scale social networks[J]. Journal of Information Science, 2015, 41(5): 686-704.

[13] Li H, Wu D, Tang W, et al. Overlapping Community Regularization for Rating Prediction in Social Recommender Systems[C]. Proceedings of the 9th ACM Conference on Recommender Systems. ACM, 2015: 27-34.

[14] Maksai A, Garcin F, Faltings B. Predicting Online Performance of News Recommender Systems Through Richer Evaluation Metrics[C]. Proceedings of the 9th ACM Conference on Recommender Systems. ACM, 2015: 179-186

[15] DING Q, LI M, YU J, et al. Group Based Multi-feature Latent Factor Model for Recommendation System in Social Network[J]. 2015.

[16] Liu X, Fulia I. Incorporating User, Topic, and Service Related Latent Factors into Web Service Recommendation[C]. Web Services (ICWS), 2015 IEEE International Conference on. IEEE, 2015: 185-192.

[17] Zhou Z, Wang B, Guo J, et al. QoS-Aware Web Service Recommendation Using Collaborative Filtering with PGraph[C]. Web Services (ICWS), 2015 IEEE International Conference on. IEEE, 2015: 392-399.

[18] Hu Y, Peng Q, Hu X, et al. Web Service Recommendation Based on Time Series Forecasting and Collaborative Filtering[C]. Web Services (ICWS), 2015 IEEE International Conference on. IEEE, 2015: 233-240. 